\title{
Créneau commercialisation des produits agroalimentaires en contexte transfrontalier : caractéristiques d'émergence et pistes pour stimuler son essor
}

\author{
Chantale Doucet \\ Consultante chercheure et doctorante en sciences sociales appliquées ${ }^{\mathrm{a}}$
}

\section{INTRODUCTION}

L'Outaouais est une région transfrontalière qui partage un bassin de vie commun avec les régions voisines de l'Ontario. Cette proximité procure à ses entrepreneurs des avantages pour la commercialisation des produits agroalimentaires. La région outaouaise se distingue en ayant accès à un marché d'exportation en circuit court. Ce marché a toujours été présent, mais l'accentuation de la demande pour les produits locaux et de qualité à Gatineau et particulièrement à Ottawa a généré de nouvelles opportunités. Du coup, on observe l'émergence d'un nouveau créneau en Outaouais avec l'apparition d'une nouvelle génération d'entreprises agricoles, de transformateurs et de plusieurs initiatives pour soutenir ce secteur. Malgré son potentiel important comme levier de développement économique régional, cette spécificité transfrontalière unique à l'Outaouais est peu étudiée, encore mal comprise, largement sousexploitée et confrontée à des contraintes majeures.

À la recherche de différentes avenues pour soutenir et stimuler le développement agroalimentaire sur le territoire, les acteurs régionaux

\section{DÉMARCHE MÉTHODOLOGIQUE}

La démarche méthodologique sur laquelle s'appuie cet article a été réalisée en deux principales étapes (voir le tableau suivant pour un résumé). La première étape avait pour principal objectif d'identifier les caractéristiques et les potentiels de la commercialisation des produits agroalimentaires en Outaouais. Pour atteindre cet objectif, nous avons mobilisé les études réalisées par le passé sur différents aspects de la filière agroalimentaire en Outaouais. Différentes données statistiques ont également été colligées, dont celles issues du recensement agricole qui permet de rendre compte de l'évolution de ce secteur ont institué une démarche inédite de réflexion afin de dégager les spécificités et les potentialités de ce nouveau créneau associé au contexte transfrontalier ${ }^{\mathrm{b}}$. Après un survol rapide de l'approche méthodologique, cet article résume le contenu de cette démarche en deux temps ${ }^{1}$. La première partie, qui vient caractériser le créneau sur la base de quatre principaux critères, aborde les dynamiques agroalimentaires en Outaouais et l'influence jouée par la proximité de l'Ontario dans le développement de la région.

Les principaux enjeux qui freinent le développement du créneau sont exposés dans la seconde partie de l'article. Des enseignements tirés de trois études de cas d'espaces transfrontaliers européens qui ont mis en place des projets pour stimuler la commercialisation agroalimentaire contribuent à dégager quelques pistes de réflexion pour éclairer la situation de l'Outaouais. Les principaux constats et réflexions sont résumés en guise de conclusion.

dans le temps et de comparer la situation régionale à celle du Québec. Pour les entreprises de transformation et celles qui ont des activités de commercialisation de produits agroalimentaires, les données sont plus difficiles à repérer. Nous avons opté pour la réalisation d'une base de données à partir des informations collectées sur différents sites Internet et validées auprès des intervenants. L'ensemble de la démarche a d'ailleurs été réalisée de concert avec un comité de représentants du milieu. Les principaux constats tirés de cette première étape ont été analysés sous l'angle de critères qui caractérisent 
le développement d'un créneau et que nous vous présentons dans la prochaine partie.

Cette première étape a non seulement contribué à identifier les forces de la filière agroalimentaire en Outaouais, mais elle a également mis en lumière des enjeux importants qui freinent le développement de la commercialisation. La deuxième étape de la démarche poursuivait donc l'objectif d'éclairer les stratégies à mettre en place pour que les entreprises agricoles et agroalimentaires de l'Outaouais puissent saisir pleinement les opportunités offertes par le marché ontarien à proximité. La réalisation d'études de cas de trois espaces transfrontaliers en Europe qui ont mis en place des stratégies et projets pour développer la commercialisation a contribué à atteindre cet objectif. Le choix de ces cas a été réalisé sur la base de la documentation disponible et de similitudes avec les enjeux et le contexte socioéconomique de l'Outaouais. Chacun des trois cas à l'étude a fait l'objet d'une revue de littérature à partir des informations disponibles sur les sites Internet et dans les revues scientifiques. La comparaison de ces études de cas a contribué à dégager des enseignements pour l'Outaouais qui sont présentés dans la dernière partie de cet article.

\section{Tableau1 - Résumé des objectifs et de l'approche méthodologique}

\begin{tabular}{|l|l|}
\hline \multicolumn{1}{|c|}{ Objectifs } & \multicolumn{1}{|c|}{ Approche méthodologique } \\
\hline $\begin{array}{l}\text { Identifier les caractéristiques, les potentiels et les } \\
\text { enjeux de la commercialisation des produits } \\
\text { agroalimentaires en Outaouais }\end{array}$ & $\begin{array}{l}\text { Revue documentaire sur différents aspects de la } \\
\text { filière agroalimentaire en Outaouais }\end{array}$ \\
\hline $\begin{array}{l}\text { Dégager des tendances et comparer la situation de } \\
\text { l'Outaouais à celle du Québec }\end{array}$ & $\begin{array}{l}\text { Analyse statistique à partir de diverses sources, } \\
\text { dont celles issues du recensement agricole }\end{array}$ \\
\hline $\begin{array}{l}\text { Identification des entreprises agricoles et } \\
\text { agroalimentaires qui ont développé au moins un } \\
\text { point de vente à l'extérieur du lieu de fabrication } \\
\text { ou de production (démarche de } \\
\text { commercialisation) }\end{array}$ & $\begin{array}{l}\text { Réalisation d'une base de données à partir des } \\
\text { informations collectées sur différents sites Internet } \\
\text { et dans différents répertoires et validées auprès des } \\
\text { intervenants }\end{array}$ \\
\hline $\begin{array}{l}\text { Éclairer les stratégies à mettre en place pour que } \\
\text { les entreprises agricoles et agroalimentaires de } \\
\text { l'Outaouais puissent saisir pleinement les } \\
\text { opportunités offertes par le marché ontarien à à } \\
\text { proximité }\end{array}$ & $\begin{array}{l}\text { Revue de littérature sur les régions transfrontalières } \\
\text { en Europe et en Amérique du Nord }\end{array}$ \\
\cline { 2 - 2 } $\begin{array}{l}\text { Choix des trois études de cas sur la base de la } \\
\text { documentation disponible et de similitudes avec les } \\
\text { enjeux de l'agriculture et l'agroalimentaire en } \\
\text { Outaouais et son contexte socioéconomique }\end{array}$ \\
\cline { 2 - 2 } $\begin{array}{l}\text { Analyse des études de cas et comparaison avec } \\
\text { l'Outaouais pour dégager des enseignements }\end{array}$ \\
\hline
\end{tabular}

\section{CARACTÉRISTIQUES ET POTENTIELS DU CRÉNEAU COMMERCIALISATION DES PRODUITS AGROALIMENTAIRES EN CONTEXTE TRANSFRONTALIER}

Les caractéristiques et potentiels du créneau commercialisation des produits agroalimentaires en contexte transfrontalier ont été examinés sous l'angle de quatre principaux critères : 1. une spécificité qui distingue le créneau; 2. le regroupement sur un même territoire d'une masse critique d'entreprises; 3 . le soutien des acteurs du milieu; 4. des effets structurants et un potentiel de croissance. Ces critères s'inspirent d'une abondante littérature scientifique sur les théories du développement territorial et sont également mobilisés dans le projet ACCORD (Action concertée de coopération régionale de développement) créé par le gouvernement du Québec afin de stimuler le développement de systèmes productifs régionaux ${ }^{2}$. 
Ce créneau est défini comme un ensemble d'entreprises qui produisent, transforment et commercialisent des produits agroalimentaires présentant des avantages uniques et concurrentiels associés au contexte transfrontalier spécifique à l'Outaouais qui lui assure un contact privilégié avec le marché et les consommateurs d'Ottawa et de l'Ontario.

\subsection{Le contexte transfrontalier, une spécificité qui procure des avantages uniques et concurrentiels à l'Outaouais}

Un créneau se démarque généralement grâce à une spécificité qui agit comme une image de marque pour la région. Située au sud-ouest du Québec et voisine d'Ottawa, la position géographique de l'Outaouais est une spécificité constamment évoquée pour caractériser le développement de la région. Tout comme l'Outaouais, plusieurs régions québécoises partagent une frontière avec d'autres provinces ou même avec les États-Unis. La frontière fait office de limite ou de barrière qui différencie deux territoires politiques. Or, la région outaouaise se distingue par son contexte transfrontalier qui implique une ouverture et une accessibilité importante entre les deux territoires adjacents qui, malgré leurs différences, ont développé une relation étroite au fil du temps.

\section{« Le passage du frontalier au transfrontalier renvoi à l'idée que le lien l'emporte sur la séparation et que des échanges structurés, organisés et durables s'effectuent sur de courtes distances de part et d'autre de la frontière ${ }^{3}$.}

Ce contexte transfrontalier unique à l'Outaouais est la spécificité qui distingue le créneau et qui procure à ses entreprises de nombreux avantages pour la commercialisation des produits agroalimentaires. La proximité géographique est un facteur concurrentiel majeur, car elle réduit les coûts associés aux transports ainsi que les contraintes liées à la périssabilité des produits agricoles ${ }^{4}$. À lui seul, le bassin de consommation Outaouais-Ottawa est estimé à plus de 1,3 million d'habitants, ce qui constitue une masse critique de consommateurs suffisamment forte pour le développement de produits agroalimentaires variés. L'intérêt pour l'achat local, les produits frais et de qualité est une tendance observée chez les consommateurs ontariens et dans le réseau des HRI (hôtels, restaurants et institutions) à Ottawa, l'un des plus importants au Canada. La population et les entreprises des deux territoires adjacents ont développé une relation étroite au fil du temps. Le secteur urbain de Gatineau, relié par un pont, est en continu avec Ottawa et une portion non négligeable de la population traverse la frontière pour occuper un emploi. En 2011, 27 \% des déplacements des Gatinois et des municipalités voisines ont pour destination la région d'Ottawa, ce qui représente 38600 déplacements ${ }^{5}$. Dans ce contexte, l'Outaouais se classe au premier rang au Québec pour l'importance de sa population bilingue (60\% comparativement à $43 \%$ pour l'ensemble du Québec) $)^{6}$. Le MAPAQ estime que près de $50 \%$ des producteurs agricoles de la région sont d'origine anglophone. Les entreprises de l'Outaouais ont développé une relation commerciale avec la clientèle ontarienne: l'agrotourisme et les marchés publics attirent les Ontariens en Outaouais; les entreprises de l'Outaouais sont présentes en Ontario par l'entremise des marchés publics, de Savourez Ottawa et dans le réseau des HRI. L'Outaouais est d'ailleurs incluse dans la « zone d'aliments locaux d'Ottawa » défini par la Ville d'Ottawa et l'organisation ontarienne Alimentation juste ${ }^{7}$. Ajoutons que 1'Ontario est souvent dans la ligne de mire des stratégies commerciales mises en place par les entreprises et les intervenants qui ont développé une connaissance fine de ce marché transfrontalier. Ces connaissances sont appuyées par une série d'études scientifiques qui ont contribué à qualifier la demande du côté ontarien dans différents créneaux. Ces études ont également révélé que la confiance et l'intérêt de l'Ontario pour les produits agroalimentaires de l'Outaouais ne sont pas une tendance passagère.

Par ailleurs, l'Ontario est identifié par le ministère de l'Agriculture, des Pêcheries et de l'Alimentation du Québec comme l'un des marchés d'exportation les plus prometteurs pour le Québec ${ }^{8}$. Pour faciliter le commerce bilatéral, les gouvernements des deux provinces ont adopté un accord de coopération dont 
l'une des visées est «d'améliorer la position concurrentielle de leurs secteurs agricoles et de la transformation agroalimentaire respectifs $»^{9}$. Le contexte transfrontalier de l'Outaouais positionne favorablement la région pour saisir les opportunités de ce marché important qui peut à la fois être qualifié de proximité et d'exportation.

\subsection{Une masse critique d'entreprises dans la filière agroalimentaire en Outaouais}

Ce caractère transfrontalier a influencé les dynamiques agricoles en Outaouais au cours des dernières années comme le révèlent quelques statistiques récentes. Sur les 29437 fermes $^{c}$ au Québec en 2011, 1167 (4\%) sont situées dans la région de l'Outaouais. La région se distingue par une majorité de fermes dans les productions animales (56\%), comparativement à $48 \%$ au Québec. L'élevage de bovins de boucherie est particulièrement important avec 369 fermes, soit $11,7 \%$ du Québec. L'Outaouais a donc été particulièrement marqué par la crise bovine au début des années 2000. Alors que le nombre de fermes se spécialisant dans l'élevage de bovins de boucherie et laitiers a diminué entre 2001 et 2011, plusieurs productions non traditionnelles dans la région connaissent un essor important insufflant un dynamisme nouveau au secteur agricole.

\section{Cette évolution est différente de celle du} Québec : plusieurs productions qui stagnent ou qui sont en décroissance au Québec connaissent, au contraire, un essor considérable en Outaouais.

(Voir les productions en gris dans le tableau suivant) :

\section{Tableau 2 - Évolution du nombre de fermes par production entre 2001 et 2011; comparaison Outaouais Québec ${ }^{10}$}

\begin{tabular}{lcc}
\hline & Outaouais & Québec \\
\hline Élevage de bovins laitiers et production laitière & $-40 \%$ & $-31 \%$ \\
\hline Élevage de bovins de boucherie & $-45 \%$ & $-39 \%$ \\
\hline Élevage de porcs & $0 \%$ & $-32 \%$ \\
\hline Élevage de volailles et production d'oeufs & $33 \%$ & $-5 \%$ \\
\hline Élevage de moutons et de chèvres & $-12 \%$ & $20 \%$ \\
\hline Autres types d'élevage & $84 \%$ & $35 \%$ \\
\hline Culture de plantes oléagineuses et de céréales & $182 \%$ & $20 \%$ \\
\hline Culture de légumes et de melons & $89 \%$ & $-11 \%$ \\
\hline Culture de fruits et de noix & $80 \%$ & $35 \%$ \\
\hline Culture en serre et en pépinière et floriculture & $39 \%$ & $1 \%$ \\
\hline Autres cultures agricoles & $86 \%$ & $45 \%$ \\
\hline
\end{tabular}

Certes, l'Outaouais avait parfois peu d'entreprises à l'origine dans ces secteurs. Il n'en demeure pas moins que cette croissance indique tout de même une transition dans les structures agricoles avec l'apparition de plusieurs entreprises orientées vers une diversité de production.

Ce dynamisme se révèle également en examinant les données sur la relève agricole ${ }^{d}$. En 2011, l'Outaouais se distingue de l'ensemble du Québec par une relève qui crée davantage de nouvelles entreprises: $52 \%$ de la relève en Outaouais ont choisi de démarrer une nouvelle entreprise agricole alors qu'au Québec, seu- lement $33 \%$ ont choisi cette voie préférant acquérir une entreprise existante en totalité ou en partie (MAPAQ, 2014) ${ }^{11}$. Les données de 2006 avaient révélé le même constat.

Dans ce contexte, deux types d'entreprises agricoles qui ont des réalités, des enjeux et des besoins fort différents se côtoient en Outaouais : les entreprises dont la commercialisation est organisée en collectif et l'apparition d'un nouveau groupe d'entrepreneurs avec commercialisation directe. Le premier groupe est assez homogène et principalement composé d'entreprises dans les productions traditionnelles (bovines et laitières). Leur structure de 
vente est organisée de longue date et desservie par des plans conjoints négociés par les fédérations. Ces agriculteurs ne sont donc pas impliqués dans les négociations avec les transformateurs et les distributeurs et dans les activités de commercialisation qui se font sous l'égide de la régie des marchés agricoles. Ces entreprises produisent principalement des produits de masse qui bénéficient d'une économie d'échelle et qui sont souvent destinés aux supermarchés.

Le deuxième groupe d'entreprises agricoles réalise la commercialisation de leur production directement et individuellement, ce qui implique une série d'actions liées à la négociation, à la vente, à la promotion et au transport des produits. Plusieurs réalisent également des activités de transformation. Ce groupe correspond essentiellement aux entreprises dont le nombre est en croissance en Outaouais, mais on y retrouve également un nombre croissant de productions traditionnelles, dans le secteur bovin ou avicole par exemple, qui ont choisi de se différencier en proposant des produits qui se distinguent par leur qualité. La propension de ce deuxième groupe qui contribue à diversifier l'agriculture régionale est directement liée au contexte de région transfrontalière.

\section{Le système agricole de l'Outaouais se module aux modes de consommation changeants de la population \\ transfrontalière qui favorisent de plus en plus les productions de qualité à proximité, ce qui permet à diverses productions de prendre un élan économique appréciable.}

Ajoutons également que l'Outaouais est une région de grands espaces agricoles qui dispose de conditions pédoclimatiques exceptionnelles adaptées à une variété d'activités agricoles, de terres de qualité à un coût compétitif et d'une excellente santé agroenvironnementale, ce qui a certes joué en faveur de ce nouveau dynamisme qui secoue actuellement le secteur agricole.

C'est dans ce contexte qu'émerge un nouveau créneau dont l'un des principaux critères est de regrouper sur un même territoire une masse critique d'entreprises dont les activités sont orientées vers la commercialisation directe de produits agroalimentaires. Sur cette base, un premier exercice a permis d'identifier 203 entreprises de l'Outaouais qui s'inscrivent dans ce créneau ${ }^{\mathrm{e}}$. Ces entreprises ont développé au moins un point de vente à l'extérieur du lieu de fabrication ou de production et sont donc déjà engagées dans un processus de commercialisation pour mettre leur produit en vente sur le marché transfrontalierf. Parmi elles, des entreprises agricoles, mais également des entreprises de transformation alimentaire à petite ou grande échelle. Ces entreprises partagent les mêmes opportunités liées au contexte géographique de l'Outaouais à proximité du marché économique de l'Ontario. Il y a quelques années à peine ce portrait n'aurait pu être réalisé, les entreprises étant trop peu nombreuses.

\subsection{Une multitude d'acteurs et d'entreprises engagés et regroupés pour appuyer la filière agroalimentaire}

Le réseautage des entreprises ainsi que l'appui des différents acteurs du milieu est un critère majeur pour favoriser le développement d'un créneau. En Outaouais, une multitude d'acteurs et d'entreprises sont engagés et regroupés pour appuyer l'essor et la consolidation de la filière agroalimentaire. Les différentes stratégies et projets mis en place ces dernières années pour soutenir l'agriculture sur le territoire démontrent que ces acteurs disposent d'une certaine capacité et d'une marge de manœuvre pour orienter le développement agricole ${ }^{12}$. Le créneau fait l'objet de transferts de connaissances avec la présence notamment du Centre de recherche et de développement technologique agricole de l'Outaouais (CREDETAO) et d'organismes de formation qui contribuent à l'avancement des connaissances et stimulent l'innovation avec le développement de nouvelles filières agroalimentaires adaptées aux réalités régionales. Ces organisations ne travaillent pas en vase clos. Elles ont développé une concertation solide notamment à travers leur participation à la Table agroalimentaire de l'Outaouais (TAO) qui a contribué à instaurer un climat convivial propice au développement d'une vision concertée arrimée à des stratégies et des actions pour soutenir la filière agroalimentaire. $\mathrm{Si}$ bien que la TAO a délaissé quelque peu son rôle de concertation afin de répondre à un besoin urgent 
dans le milieu avec la mise en place de servicesconseils à la commercialisation.

Mentionnons d'autres démarches de concertation et de partenariat dans le milieu qui apportent un soutien au créneau: initiatives d'appui à l'agriculture biologique et à la relève agricole, dont la plate-forme agricole biologique qui fait figure de modèle au Québec; des circuits de commercialisation alternatifs sont apparus aux côtés des commerces au détail de produits alimentaires et de la restauration qui constituent déjà une force importante sur le territoire; initiatives agrotouristiques, marchés publics, marché de solidarité, foire gourmande et activités promotionnelles sont quelques exemples d'initiatives qui soutiennent la commercialisation directe des produits agroalimentaires.

\subsection{Des effets structurants pour l'Outaouais avec un potentiel de croissance important}

Finalement, un créneau doit apporter un effet structurant pour la région tout en étant appelé à jouer un rôle majeur dans l'économie nordaméricaine et mondiale. À cet égard, la capacité d'innovation et le dynamisme des entreprises et organisations qui s'inscrivent dans le créneau en Outaouais, ainsi que les opportunités engendrées par la proximité du marché ontarien, ont un effet levier structurant sur la région. La valeur du capital agricole régional et les revenus agricoles bruts sont en croissance. De nouvelles entreprises démarrent, des exploitations existantes prennent de l'ampleur. Les activités de transformation sont en croissance chez les producteurs agricoles dont plusieurs se sont dotés d'équipements automatisés augmentant la compétitivité et la performance des entreprises. Le succès d'entreprises dans la transformation, comme la nouvelle laiterie dont les activités ont été relancées en 2010 dans un domaine en perte de vitesse au Québec, a un effet d'entraînement sur le secteur de la transformation. L'offre dans plusieurs secteurs agroalimentaires ne suffit pas à la demande du marché à proximité, ce qui laisse entrevoir des possibilités de développement important. Pour certaines entreprises, le bassin de consommateurs lié au contexte transfrontalier a eu un effet levier qui a permis à leur entreprise d'atteindre d'autres marchés au Canada et à l'étranger.

\section{ANALYSE DES ENJEUX DE L'OUTAOUAIS À LA LUMIÈRE DE SIX ENSEIGNEMENTS TIRÉS D'ÉTUDES DE CAS TRANSFRONTALIÈRES}

Malgré un essor des entreprises agricoles à commercialisation directe, ce créneau est confronté à des défis importants. Plusieurs démarches de commercialisation sont encore embryonnaires malgré le potentiel important du marché transfrontalier. Les entreprises sont souvent de petites tailles et peu d'entre elles commercialisent leurs produits dans les supermarchés. Le développement d'une filière et la commercialisation des produits agroalimentaires exigent également de consolider et de maximiser les différents maillons de la chaîne : production, transformation, collecte, transport, distribution, gestion des ventes, activités de promotion et marketing, etc. Les entrepreneurs de l'Outaouais travaillent souvent de façon individuelle pour réaliser l'ensemble de ces étapes, ce qui nuit à la compétitivité de la filière agroalimentaire.

En outre, si la frontière est inexistante pour une grande majorité de la population, la situation est bien différente pour les éleveurs qui sont, rappelonsle, le groupe d'agriculteurs le plus nombreux en
Outaouais. Seules les viandes produites dans des abattoirs et des établissements de transformation accrédités auprès du gouvernement fédéral peuvent traverser la frontière entre l'Outaouais et l'Ontario. L'Outaouais compte trois abattoirs qui sont opérationnels dont un seul fédéral qui est toutefois privé avec une capacité limitée. La majorité des éleveurs n'ont donc pas accès à un abattoir fédéral. Les exigences qui permettraient d'enlever cette barrière sont extrêmement élevées et les projets qui ont été mis en place par les acteurs du milieu pour y répondre au cours des dernières années ont connu des obstacles majeurs et ont freiné certains élans de l'Outaouais pour conquérir le marché ontarien. Dans ce contexte, comment consolider davantage le positionnement des produits de l'Outaouais sur le marché ontarien? En guise de réponse, l'étude de trois régions transfrontalières qui vivent des enjeux similaires a apporté quelques pistes de réflexion.

La coopération transfrontalière existe depuis longtemps en Europe afin de faire face à certains 
problèmes locaux comme le transport et les infrastructures. Elle s'est toutefois accentuée depuis les années 1990 avec le soutien financier de l'Union européenne qui valorise le développement des projets transfrontaliers dans différents domaines ${ }^{13}$. Phénomène relativement récent, la littérature sur les régions transfrontalières reste limitée, d'autant plus si on y ajoute l'agriculture et l'agroalimentaire comme secteur spécifique. Une douzaine d'espaces transfrontaliers en Europe et en Amérique du Nord, qui ont amorcé une coopération ou du moins une réflexion en lien avec l'agriculture transfrontalière, ont tout de même été répertoriés. Parmi ces cas, trois ont retenu notre attention. Même s'ils sont très différents, ces cas s'appuient tout de même sur plusieurs bases communes que nous avons regroupées en six principaux enseignements. Ces enseignements sont mobilisés afin d'éclairer les réflexions et les pistes d'action en Outaouais en lien avec les enjeux de commercialisation, d'adaptation des mécanismes législatifs et règlementaires et le développement de la filière agroalimentaire.

\subsection{Développer un historique de coopération transfrontalière}

Les cas étudiés en Europe visent à résoudre une problématique partagée par l'ensemble du territoire transfrontalier: pression urbaine et valorisation de l'agriculture pour l'agglomération franco-valdo-genevoise; consolidation et développement de la filière des plantes à parfum, aromatiques et médicinales pour l'espace méditerranéen; enjeu lié à la mise en place d'un abattoir en Cerdagne et appui au développement de la filière bovine.

\section{La coopération transfrontalière est vue comme un levier pour la dynamisation des milieux agricoles et poursuit l'objectif d'un développement plus équilibré et cohérent entre les deux territoires en tentant de briser l'isolement économique et la fragmentation tout en renforçant les atouts que procure leur position géographique.}

Elle permet, dans certains cas, de faire face à des problèmes locaux qui n'ont pas de frontières politico administratives; ou, à l'inverse, la coopération transfrontalière permet de réfléchir aux problématiques engendrées par la frontière et sa législation et qui freinent le développement des territoires limitrophes. Pour que la coopération transfrontalière soit possible, le projet doit répondre à un intérêt partagé et une volonté commune entre les deux territoires qui sont affectés par des enjeux similaires.

Dans les cas étudiés, les projets de coopération transfrontalière n'ont pas émergé du jour au lendemain. Il s'agit plutôt d'une démarche qui s'inscrit dans le temps et qui débute par de petites initiatives de collaboration, ce qui renforce la coopération et l'identité du territoire transfrontalier et contribue au succès d'un projet de plus grande envergure.

Malgré un bassin de vie commun en Outaouais, les différentes organisations de développement et élus travaillent en vase clos, car le Québec et l'Ontario ont des structures politiques et institutionnelles distinctes. Dans ce contexte, les acteurs de l'Outaouais et de l'Est ontarien n'ont pas un historique de collaboration très important que ce soit dans le secteur agricole ou dans d'autres secteurs $^{\mathrm{g}}$. Sans cet historique de coopération, la mise en place d'un projet transfrontalier d'envergure ferait face à des obstacles majeurs liés à l'absence de développement de liens de confiance dans le passé et à une méconnaissance des réalités de chacun.

Des projets innovateurs axés sur la coopération transfrontalière ont toutefois émergé dans les dernières années. Dans le secteur agroalimentaire, la Foire gourmande Outaouais-est ontarien qui connait un succès enviable depuis 2010 est une expérience pionnière pour soutenir l'essor des produits agroalimentaires qui démontre que cette collaboration est non seulement possible, mais rejoint l'intérêt des entreprises et acteurs des deux côtés de la rivière. Cette collaboration a été facilitée par la présence d'organisations de développement (Sociétés d'aide au développement des collectivités; Centre local de développement) de chaque côté de la rivière qui partagent une identité organisationnelle commune. Mentionnons également l'accentuation des collaborations des entreprises et intervenants de l'Outaouais avec certaines organisations du côté d'Ottawa comme Savourez Ottawa. Ajoutons que la population de certains 
territoires, comme le Pontiac, partage également des liens identitaires forts avec la région ontarienne voisine, ce qui facilite la mise en place d'initiatives communes entre les deux territoires.

Ce type de collaboration qui rallie le territoire transfrontalier pourrait-il être pertinent pour faire face aux enjeux de commercialisation agricole identifiés en Outaouais? Il s'agit d'une piste de réflexion très fertile sur laquelle les acteurs de l'Outaouais se sont peu penchés jusqu'à maintenant et qui pourrait mener à des initiatives innovatrices importantes pour développer le secteur agroalimentaire. Par exemple, les efforts des acteurs de l'Outaouais pour diminuer les contraintes commerciales liées à la frontière ont été vains jusqu'à maintenant se heurtant aux exigences des paliers gouvernementaux supérieurs. À ce titre, les études de cas démontrent que pour donner de l'importance à la démarche et faire des avancées, les acteurs doivent afficher ouvertement une volonté commune de part et d'autre de la frontière. La mise en place d'une activité d'échange regroupant les acteurs des deux côtés de la rivière pourrait redonner un nouveau souffle à ce dossier et contribuer à identifier des pistes de solution qui n'avaient jusque-là pas été envisagées. Du moins, ce type d'initiative serait une bonne base pour comprendre les enjeux agroalimentaires de chacun des territoires qui restent méconnus.

\subsection{Mettre en place des activités d'échange et de réseautage impliquant une diversité d'acteurs}

Les études de cas ont mis en exergue l'importance de mettre en place un réseau d'échange et de diffusion des informations entre les différents acteurs pour stimuler l'innovation et dynamiser la filière agroalimentaire. Ces activités ont permis de rapprocher les acteurs des deux côtés de la frontière, mais également à l'intérieur même des territoires. La présence de cinq principaux groupes s'est avérée essentielle pour le succès des projets transfrontaliers. 1. Dans tous les cas, les milieux agricoles ont été fortement impliqués dans l'orientation des projets et dans la mise en œuvre, l'objectif étant qu'ils bénéficient du projet pour développer leur entreprise, ce qui génère des retombées pour l'ensemble de la filière. 2 . Les entreprises de transformation sont des partenaires majeurs pour développer une filière performante. 3. L'implication des acteurs locaux (institutions de développement local, élus locaux, etc.) est un élément incontournable des cas étudiés. 4. Les Centres de recherche sont des acteurs clés mobilisés dans la coopération transfrontalière pour stimuler l'innovation à travers le transfert de connaissance.

En Outaouais, les activités transfrontalières sont presque inexistantes entre l'Outaouais et sa voisine comme nous l'avons évoqué au point précédent. Les organisations locales de développement sont toutefois très impliquées dans le soutien de l'agriculture et de l'agroalimentaire, ce qui constitue une force importante sur laquelle il faut continuer de miser dans l'avenir tout comme l'implication et le développement des Centres de recherche comme le CRÉDÉTAO reconnu pour générer l'innovation nécessaire au développement. En outre, les entreprises qui commercialisent directement leurs produits se rencontrent principalement dans les marchés publics, MSRO, évènements comme la Foire Gourmande, journée thématique, etc. Ces initiatives sont-elles suffisantes pour réseauter adéquatement les entreprises? Étant donné que le réseautage entre les différentes entreprises constitue un facteur important d'innovation et de développement, il faut se questionner davantage et sonder l'intérêt des entrepreneurs pour participer à un réseau d'échange et de diffusion des informations entre les entreprises axé sur les différents besoins et réalités.

5. Dans les cas étudiés, la coopération transfrontalière a d'abord débuté sur le terrain avec les acteurs locaux, mais sans exception, l'accompagnement de l'État et notamment de l'Union Européenne a été un incitatif important pour propulser cette coopération à une autre étape avec des projets majeurs. Cette légitimation institutionnelle a permis de mettre en place des conditions propices au développement transfrontalier. Ce contexte n'est pas le même au Canada, où les gouvernements canadien, ontarien et québécois ont peu favorisé le développement de liens entre 1'Outaouais et l'Ontario. Étonnamment, la situation est différente pour la frontière Canada/États-Unis qui suscite un intérêt grandissant de la part des gouvernements suite à la signature de l'Accord de libre-échange nord- 
américain (ALENA) qui vise à éliminer les obstacles au commerce et faciliter la circulation transfrontalière des biens et des services entre les territoires. Le Canada a d'ailleurs mis en place un projet de recherche sur la portée et la nature des relations régionales transfrontalières qui « montre plus que jamais la nécessité d'adopter une optique régionale transfrontalière pour reconnaitre et comprendre les liens coopératifs croissants et la participation grandissante des acteurs régionaux et des intervenants locaux à la résolution pratique de problèmes communs dans les régions frontalières du Canada et des États-Unis, et pour mieux réagir en conséquence $»^{14}$.

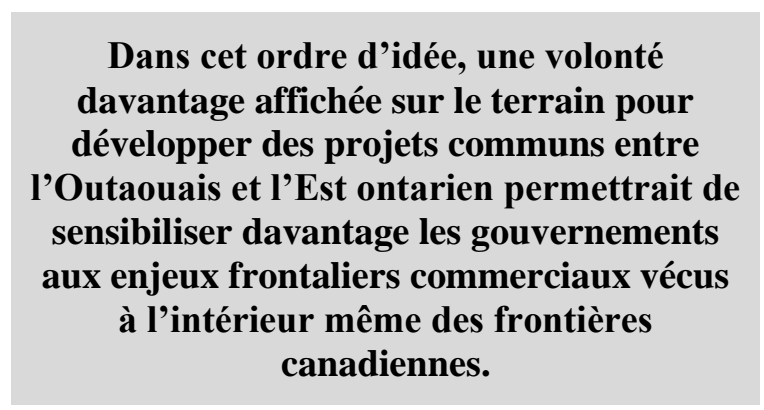

\subsection{Mutualiser les outils et les services}

Afin d'assurer une meilleure rentabilité économique, accentuer la concurrence et atteindre de nouveaux marchés, les trois études de cas présentées ont misé sur la mutualisation d'outils et de services sous diverses formes. En Outaouais, plusieurs outils et services ont été mutualisés dans les dernières années afin d'appuyer la commercialisation. Le marché de solidarité régionale (MSRO) regroupe l'offre de produits agroalimentaires locaux. Les producteurs agricoles se sont regroupés au sein d'une Coopérative d'utilisation de matériel agricole (CUMA) afin de partager la machinerie agricole et divers équipements. Le modèle coopératif est également bien présent en Outaouais dans le domaine agroalimentaire : Coop Agrodor, MSRO, Marché public de la Petite-Nation, Coopérative des consommateurs de la laiterie de l'Outaouais, Les Serres de l'Outaouais, Tierra coop, etc. Le Parcours Outaouais Gourmet regroupe les entreprises qui ont développé une stratégie commerciale en agrotourisme et la campagne et le site Internet Croquez l'Outaouais font la promotion des produits agroalimentaires régionaux sous une même vitrine.

Si certains maillons de la filière agroalimentaire ont fait l'objet d'actions concrètes pour maximiser le rendement, d'autres maillons restent faibles. L'Outaouais a développé plusieurs marchés publics au cours des dernières années. La majorité de ces marchés sont toutefois de petites tailles avec peu de producteurs, ce qui engendre des difficultés pour attirer une clientèle importante et régulière. Ces services pourraient être davantage mutualisés afin d'améliorer l'offre et la demande. La transformation des produits agroalimentaires est principalement réalisée de façon individuelle en Outaouais dupliquant les coûts pour la réalisation de cette étape. Un nombre croissant d'agriculteurs se sont automatisés au cours des dernières années et certains disposent de cuisines de transformation performantes. Or, ces cuisines ne sont pas utilisées au maximum de leur capacité et demeurent des équipements qui pourraient être mutualisés davantage. La distribution et la commercialisation des produits sont également réalisées en majeure partie individuellement. Rappelons toutefois que l'Outaouais est une vaste région. Les distances entre les entreprises sont importantes accentuant les difficultés pour mutualiser les outils et services sur le territoire. Finalement, mentionnons que l'abattoir est vu dans les études de cas comme un outil mutualisé et un maillon essentiel au développement d'une filière. Dans l'un des cas étudiés, le regroupement du service de part et d'autre de la frontière a permis de rassembler une masse critique d'éleveurs ainsi que le financement nécessaire à la construction d'un abattoir moderne adapté aux nouvelles normes européennes.

\subsection{Accentuer l'apport de connaissances, le transfert et l'accompagnement des entreprises}

L'apport de connaissances est souvent la première étape qui a contribué à amorcer les projets de coopération transfrontaliers. Certaines études ont dressé un état des lieux des réalités socioéconomiques des différents territoires alors que d'autres ont porté sur un enjeu pointu. Plusieurs études sur l'agriculture et l'agroalimentaire ont été réalisées au cours des dernières années en Outaouais. Les acteurs ont ainsi une 
bonne connaissance des réalités socioéconomiques et des enjeux, ce qui a contribué à orienter les actions à prioriser sur le territoire. Une série d'études scientifiques est également venue qualifier la demande du côté ontarien dans différents créneaux. Certaines filières ont également fait l'objet d'études spécifiques afin d'analyser leur potentiel.

Puisque les réalités et les actions évoluent constamment sur le territoire, l'apport de connaissance n'est jamais terminé. Ainsi, en lien avec les enjeux commerciaux qui nous préoccupent dans le cadre de cette étude, il existe sans doute certaines lacunes au niveau des connaissances qui nuisent à la compréhension de certains phénomènes. Mentionnons, à titre d'exemple, les besoins et les aspirations des entreprises sur le territoire concernant la commercialisation ou encore les problématiques concernant la frontière. L'apport de connaissance sur ces sujets spécifiques pourrait rejoindre l'intérêt des acteurs de l'Outaouais, mais également ceux de l'Est ontarien.

\subsection{Valoriser les spécificités territoriales et les marchés locaux}

En Outaouais, plusieurs entreprises ont orienté leurs stratégies commerciales vers le marché local, compris ici comme l'ensemble du territoire transfrontalier incluant l'Outaouais et le territoire limitrophe de l'Ontario. Les études de cas viennent confirmer qu'il s'agit d'un levier important pour développer la filière agroalimentaire. Toutefois, plusieurs opportunités du marché local restent encore sous-exploitées et échappent aux entreprises de l'Outaouais.

Les territoires transfrontaliers ont également valorisé les caractéristiques identitaires et qualitatives des produits. Ces démarches peuvent inspirer l'Outaouais pour valoriser davantage les atouts de ses produits agroalimentaires. Plusieurs activités promotionnelles qui tablent sur les produits régionaux ont toutefois été mises en place ces dernières années auprès de la clientèle régionale et ontarienne. Les études de cas ont démontré que ces démarches collectives ont une portée importante pour développer la commercialisation.

\subsection{Appuyer les stratégies de développement durable}

Finalement, les projets étudiés n'hésitent pas à mettre de l'avant le caractère durable et multifonctionnel de l'agriculture. Pour pérenniser l'agriculture qui est confrontée à des pressions urbaines importantes, l'agglomération franco-valdo-genevoise appuie ses stratégies sur l'importance du rôle des espaces agricoles dans la qualité de vie et la préservation de l'environnement et des paysages sur le territoire. Dans la filière des plantes à parfum, aromatiques et médicinales, le développement de techniques d'économie d'énergie diminue les coûts liés à la transformation générant une valeur ajoutée et un avantage concurrentiel aux produits. L'abattoir de proximité en Cerdagne contribue à réduire les déplacements et la consommation de carburant, favorisant la préservation de l'environnement et du bien-être animal. En contribuant au maintien de l'agriculture sur le territoire, il participe du même coup à la préservation des paysages et à la qualité de vie. Mentionnons également que certains abattoirs valorisent leurs déchets, ce qui engendre des bénéfices environnementaux et économiques.

En Outaouais, il y a peu de problématiques environnementales liées à l'agriculture. Les élevages et les cultures sont à petites échelles; il n'y a pas de mégaporcherie; les plantes fourragères pérennes couvrent $85 \%$ des superficies cultivées; $14,2 \%$ des pâturages naturels au Québec sont en Outaouais; l'agriculture est en équilibre écologique avec les boisés, milieux naturels et cours d'eau. Alors que dans d'autres régions les entreprises agricoles subissent de fortes pressions pour changer les pratiques en matière d'environnement, l'Outaouais se distingue avec un bilan environnemental enviable. Sans compter que l'agriculture est également un facteur de pérennité socioéconomique pour plusieurs communautés. Dans ces conditions, l'agriculture outaouaise et ses produits peuvent être qualifiés de durables. Méconnus et peu valorisés, ces atouts pourraient être davantage mobilisés pour appuyer la commercialisation, car développement durable et territoire transfrontalier vont de pairs. 


\section{CONCLUSION}

Dans cet article, nous avons démontré l'émergence d'un nouveau créneau économique composé d'une masse critique d'entreprises dont les activités sont orientées vers la commercialisation directe de produits agricoles destinés au marché transfrontalier. Ce marché exceptionnel est une force unique à l'Outaouais qui procure des avantages concurrentiels à sa filière agroalimentaire. Le savoir-faire, l'expertise et l'accompagnement des différents acteurs du milieu ainsi que les projets et stratégies qui soutiennent la transformation et la commercialisation de produits agroalimentaires offrent une atmosphère propice au développement du créneau. Malgré son effet levier structurant pour l'ensemble de l'Outaouais, ce créneau en émergence est toutefois confronté à des défis importants. L'analyse d'études de cas de territoires transfrontaliers qui ont mis en place des initiatives associées à la commercialisation agroalimentaire a démontré les bénéfices que pourrait engendrer l'accentuation de la coopération entre l'Outaouais et les régions ontariennes voisines. Les barrières règlementaires qui freinent l'accès au marché frontalier pour les produits carnés et alcoolisés est un enjeu propice pour amorcer une coopération transfrontalière que ce soit dans la réalisation d'une étude commune, la mise en place d'une activité d'échange avec les Ontariens ou encore pour sensibiliser les gouvernements aux enjeux frontaliers commerciaux vécus entre l'Outaouais et l'Ontario. Les études de cas ont également démontré l'importance de mettre en place des outils mutualisés pour consolider les différents maillons de la filière agroalimentaire (regroupement de l'offre, transformation et distribution) et consolider le positionnement des produits de l'Outaouais sur le marché ontarien. L'intégration $\mathrm{du}$ caractère durable et multifonctionnel de l'agriculture dans les stratégies transfrontalières est également un élément sur lequel il faut miser pour innover et créer des avantages comparatifs.

En somme, si plusieurs conditions propices au développement du créneau sont en place, d'autres restent à instaurer afin de valoriser pleinement son potentiel. Rappelons que le présent article s'inscrit dans une démarche de valorisation instituée par les acteurs régionaux afin d'alimenter les connaissances sur ce créneau et orienter les actions à prioriser sur le territoire. Pour ces acteurs, la valorisation de ce créneau apparait comme l'une des avenues sur laquelle il faut miser pour stimuler le développement et l'innovation de l'Outaouais.

\section{BIBLIOGRAPHIE}

${ }^{1}$ Ces deux parties s'appuient sur les études suivantes: Doucet, Chantale (2014). Étude d'étalonnage: comparaison des dynamiques agricoles et agroalimentaires de trois régions transfrontalières; pistes de réflexion pour l'Outaouais, Rapport réalisé par Projets Territoires pour la Table agroalimentaire de l'Outaouais et ses partenaires, 78 pages; Doucet, Chantale (2013). Étude de tendances et d'opportunités. Commercialisation des produits agroalimentaires en contexte transfrontalier. Région de l'Outaouais, Rapport réalisé par Projets Territoires pour la Table Agroalimentaire de l'Outaouais et ses partenaires, $82 \mathrm{p}$. ${ }^{2}$ Créneaux d'excellence. Site du ministère de l'Économie, innovation et Exportation: https://www.economie.gouv.qc.ca/objectifs/informer/cre neaux-dexcellence/

${ }^{3}$ Reitel, Bernard et Patricia Zander (2004). Espace transfrontalier. 3 pages. Hypergeo, http://www.hypergeo.eu/spip.php?article207 consulté le 9 septembre 2013.

${ }^{4}$ Gallaud, Delphine, Michel Martin, Sophie Reboud et Corinne Tanguy (2012). Proximités organisationnelle et géographique dans les relations de coopération : une application aux secteurs agroalimentaires, Géographie, économie, société, 2012/3 (Vol. 14), P.261 à 285.

${ }^{5}$ Malatest \& Associates (2013). 2011 NCR Household Origin-Destination Survey. Summary of Results, 150 p.

${ }^{6}$ Statistique Canada, Recensement 2011.

${ }^{7}$ Alimentation juste (2012). L'Accès aux aliments sains et aux aliments locaux - Planification et zonage, http://www.justfood.ca/foodforall/fr/weblinks/laccesaux-aliments-planification-et-zonage/ consulté le 4 octobre 2013.

${ }^{8}$ MAPAQ - Ministère de l'Agriculture, des Pêcheries et de l'Alimentation, Direction du développement des marchés extérieurs et Bureau du Québec à Toronto. (2012). Regard sur le marché de l'Ontario -Toronto.

${ }^{9}$ Gouvernement du Québec et de l'Ontario (2011 - mise à jour du document de 2009). Accord de commerce et de coopération entre le Québec et l'Ontario, 103 p.

${ }^{10}$ Statistique Canada, Recensement 2001 et 2011, compilation Doucet, 2013

${ }^{11}$ MAPAQ (2014). Portrait de la relève agricole au Québec 2011. Gouvernement du Québec, 55 p. + annexes. 
${ }^{12}$ Doucet, Chantale (2015 - à paraitre). Valorisation des spécificités agricoles régionales par les acteurs publics territoriaux : des mouvements ascendant et descendant jonchés d'embuches, L'entrepreneuriat public : soutenir l'innovation bioalimentaire, ouvrage sous la direction de M.J. Fortin et Mario Handfield, Université du Québec à Rimouski.

${ }^{13}$ Mettan, Nicolas (2003). Enjeux et perspectives de la coopération transfrontalière dans le domaine de l'aménagement du territoire : l'exemple de la Suisse romande, Revue d'Économie Régionale \& Urbaine 5/ 2003 (décembre), p. 951-972

${ }^{14}$ Gouvernement du Canada (2008). L'émergence des régions transfrontalières entre le Canada et les ÉtatsUnis. Récolter la promesse et la valeur publique des relations régionales transfrontalières, Rapport final, $82 \mathrm{p}$.

\section{NOTES}

${ }^{a}$ Chantale Doucet est consultante chercheure. Le présent article est un condensé de deux études réalisées pour le milieu. Une recherche doctorale sur les dynamiques territoriales en agriculture est également venue bonifier l'analyse.

${ }^{b}$ Les acteurs régionaux qui ont participé à cette démarche sont la Table agroalimentaire de l'Outaouais, le ministère de l'Agriculture, des Pêcheries et de l'Alimentation du Québec, le ministère de l'Économie, de l'Innovation et des Exportations, la Conférence régionale des élus de l'Outaouais, Emploi Québec Outaouais. Développement économique Canada et la Ville de Gatineau ont également été des partenaires financiers importants pour la réalisation de l'étude d'étalonnage.

${ }^{c}$ Les statistiques de cette partie sont tirées principalement des recensements agricoles qui ont lieu à tous les cinq ans.

${ }^{\mathrm{d}} \mathrm{Ce}$ recensement s'adressait à l'ensemble des agriculteurs et agricultrices du Québec âgé de moins de 40 ans et possédant au moins $1 \%$ des parts d'une entreprise agricole.

'Il n'existe pas de statistiques au Québec qui rassemble ces pratiques. Diverses sources ont donc été utilisées pour répertorier ces entreprises.

fPar exemple, une boucherie ou un restaurant qui vendent uniquement sur place ne sont pas répertoriés dans la liste à moins que leurs produits soient également commercialisés chez d'autres détaillants. De même, les entreprises agricoles qui vendent leur produit uniquement à la ferme sont également exclues. Elle recèle toutefois un potentiel important pour développer le créneau.

gMentionnons tout de même la présence de la Commission de la capitale nationale (CCN), une société d'État du gouvernement du Canada dont le mandat de conservation et d'embellissement de la région de la capitale du Canada couvre les territoires d'Ottawa et de Gatineau ainsi que les municipalités limitrophes. 